INPLASY

PROTOCOL

To cite: Wu et al. Laparoscopic versus robot techniques for the choledochal cyst: a metaanalysis. Inplasy protocol 202190082. doi:

10.37766/inplasy2021.9.0082

Received: 23 September 2021

Published: 23 September 2021

Corresponding author:

Qiao Wu

2775310211@qq.com

Author Affiliation:

Affiliated Hospital of North

Sichuan Medical College.

Support: 20PJ147.

Review Stage at time of this submission: Data analysis.

Conflicts of interest:

None declared.

\section{Laparoscopic versus robot techniques for the choledochal cyst:} a meta-analysis

\author{
Wu, Q1; Zhao, D2; Wang, C³.
}

Review question / Objective: Recently, several studies have compared the postoperative outcomes in patients with choledochal cyst who underwent robotic laparoscopicassisted choledochocystectomy to patients with choledochal cyst who underwent laparoscopic choledochocystectomy. But the results remained controversies. Therefore, we conducted this meta-analysis to compare the postoperative outcomes between robotic techniques and laparoscopic approach for the management of choledochal cyst.

Information sources: The Cochrane Central Search Library, PubMed, and Embase were used to search for related studies that compared the outcomes of laparoscopic surgery for choledochal cyst to robotic technique.

INPLASY registration number: This protocol was registered with the International Platform of Registered Systematic Review and Meta-Analysis Protocols (INPLASY) on 23 September 2021 and was last updated on 23 September 2021 (registration number INPLASY202190082).

\section{INTRODUCTION}

Review question / Objective: To compare the postoperative outcomes between robotic techniques and laparoscopic approach for the management of choledochal cyst through a meta-analysis.

Condition being studied: Recently, several studies have compared the postoperative outcomes in patients with choledochal cyst 
who underwent robotic laparoscopicassisted choledochocystectomy to patients with choledochal cyst who underwent laparoscopic choledochocystectomy. But the results remained controversies. Therefore, we conducted this meta-analysis to compare the postoperative outcomes between robotic techniques and laparoscopic approach for the management of choledochal cyst.

\section{METHODS}

Participant or population: Patients with choledochal cyst.

Intervention: Robotic techniques.

\section{Comparator: Laparoscopy.}

Study designs to be included: Retrospective comparative study.

Eligibility criteria: (1) studies which compared robotic techniques to laparoscopic surgery for the treatment of choledochal cyst; (2) relevant data could be accessible in robot group and laparoscopy group; (3) studies written in English; (4) studies with full text.

Information sources: The Cochrane Central Search Library, PubMed, and Embase were used to search for related studies that compared the outcomes of laparoscopic surgery for choledochal cyst to robotic technique.

Main outcome(s): The main outcomes include blood loss, postoperative complications, operative time and length of hospital stay.

Quality assessment / Risk of bias analysis: The Newcastle-Ottawa Scale was applied for evaluating the quality of each included study.

Strategy of data synthesis: Odds ratio (OR) and its $95 \%$ confidential interval $(\mathrm{Cl})$ were used to assess dichotomous data, while weight mean differences (MDs) were used to assess continuous data.
Subgroup analysis: Due to the small number of original literatures included, we did not conduct subgroup analysis.

Sensitivity analysis: Due to the small number of original literatures included, we did not conduct subgroup analysis.

Country(ies) involved: China.

Keywords: robotic techniques; laparoscopy; choledochal cyst; metaanalysis.

Contributions of each author:

Author 1 - Qiao Wu - Writing the manuscript.

Email: 2775310211@qq.com

Author 2 - Dan Zhao - Data analysis.

Email: zd_5404@163.com

Author 3 - Cheng Wang - Coming up with idea.

Email: wangchengemail@126.com 